Thais Scaglione, Valkiria Pedri Fialkowski, Emanuela

Lima Silveira, Aguinaldo Dos Santos *

\title{
Estado da Arte sobre 0 uso de Big Data no Design: Perspectiva de Sistemas Produtos + Serviços Sustentáveis
}

Thais Scaglione é Mestranda em Design pela UFPR, especialista em Gestão de Tendências/Coolhunting e bacharel em Desenho Industrial com Habilitação em Programação Visual pela PUCPR. Sócia-fundadora do Estúdio Sem Dublê e pesquisadora do Núcleo de Design \& Sustentabilidade da UFPR nas áreas de Data-Driven Design \& Sustainable Behaviour. <thais.scaglione@hotmail.com> ORCID: 0000-0003-1483-9512

Valkiria Pedri Fialkowski é Mestre e doutoranda em Design pela UFPR, possui MBA em Marketing pela FGV e bacharelado em Desenho Industrial pela UFPR. Sócia-fundadora da empresa Arbo Design e pesquisadora do Núcleo de Design \& Sustentabilidade da UFPR nas áreas de Data-Driven Design \& Sustainable Behaviour.

<val.pedri@gmail.com> ORCID: 0000-0002-9030-7764
Resumo Desenvolver ou aprimorar Sistemas Produtos+Serviços (PSSs) de forma efetiva e sustentável que atendam às necessidades e expectativas dos usuários e stakeholders é um grande desafio. Neste cenário, o big data tem se mostrado como uma importante fonte para apoiar a tomada de decisão e identificar padrões, como o de comportamento. 0 objetivo deste artigo é apresentar uma análise crítica, exploratória e qualitativa da literatura, sobre estudos de big data para o Design de Sistemas Produto+Serviço Sustentável. Por meio do método de Revisão Bibliográfica, foi possível identificar lacunas e ênfases acerca dos temas abordados. Os resultados apontam para um uso incipiente do big data no campo do Design para a Sustentabilidade. Existe um baixo índice do uso dos dados para análises preditivas em Design e PSS. Enfatiza-se, desse modo, a necessidade de produção acadêmica e desenvolvimento de estratégias e modelos para viabilização do trabalho interdisciplinar do designer.

Palavras chave PSS Design, Big Data, Estado da Arte, Tecnologias da Informação, Sustentabilidade. 


\section{State of the Art on the use of Big Data in Design: Perspective of Sustainable Products+Services Systems}

Emanuela Lima Silveira é Doutoranda em Design (PPGDesign - UFPR), Mestre em Engenharia Mecânica (PPGEM-UTFPR) e Bacharel em Design (DADIN-UTFPR). Foi uma das fundadoras na Empresa Junior de Design da UTFPR (Estalo-DADIN). Atuou como bolsista de Iniciação Científica PIBIC CNPq. Atualmente é pesquisadora do Núcleo de Design \& Sustentabilidade da UFPR (Bolsista Capes), na área de Criatividade para Sistemas Complexos \& Sistema Produto+Serviços Sustentáveis, seus últimos projetos de pesquisa foram realizados com a empresa Multinacional Tigre e com a ÖUS Streetwear.

<manuhsilveira@gmail.com>

ORCID: 0000-0003-2315-8188

Aguinaldo Dos Santos é Mecânico de Automóveis (SENAI), Engenheiro Civil (UFPR), Mestre em Engenharia Civil (UFRGS), Doutor em Ambiente Construído (SALFORD / UK), Pós-doutorado em Design para Sustentabilidade (POLIMI / Itália). Coordenador do Núcleo de Design \& Sustentabilidade da Universidade Federal do Paraná. Possui “Bolsa de Produtividade Nível 2" do CNPq (Agência Brasileira de Financiamento) desde 2004. Membro do Conselho de Administração do Centro Brasileiro de Design (CBD). Membro do conselho editorial de várias revistas. <asantos@ufpr.br> ORCID: 0000-0002-8645-6919
Abstract Developing Products + Services Systems (PSSs) effectively and sustainable that meet the needs and expectations of each user and stakeholder is a major challenge. In this scenario, big data has proved to be an important source of data to support decision making and identify patterns, such as behavior. The objective of this article is to present a critical, exploratory analysis of the literature, on big data studies for the Design of Sustainable Product + Service Systems. Through the Bibliographic Review method, it was possible to identify gaps and emphases about the topics covered. The results point to an incipient use of big data in the field of Design for Sustainability. There is a low rate of data use for predictive analysis in Design and PSS. In this way, the need for academic production and the development of strategies for the feasibility of the interdisciplinary work of the designer are emphasized.

Keywords PSS Design, Big Data, State of art, Information Technologies, Sustainability.

\section{Estado del arte en el uso de Big Data en el diseño: Perspectiva de los sistemas de productos y servicios sostenibles}

Resumen Desarrollar Sistemas de Productos + Servicios (PSS) de manera eficaz y sostenible que satisfagan las necesidades y expectativas de cada usuario y parte interesada es un gran desafío. En este escenario, el big data ha demostrado ser una fuente importante de datos para apoyar la toma de decisiones e identificar patrones, como el comportamiento. El objetivo de este artículo es presentar un análisis crítico y exploratorio de la literatura sobre estudios de big data para el Diseño de Sistemas de Producto + Servicio Sostenibles. A través del método de Revisión Bibliográfica, fue posible identificar vacíos y énfasis sobre los temas tratados. Los resultados apuntan a un uso incipiente de big data en el campo del Diseño para la Sostenibilidad. Hay una baja tasa de uso de datos para análisis predictivo en Diseño y PSS. De esta forma, se enfatiza la necesidad de producción académica y el desarrollo de estrategias para la viabilidad del trabajo interdisciplinario del diseñador.

Palabras clave Diseño de PSS, Big Data, Estado del arte, Tecnologías de la Información, Sostenibilidad. 


\section{Introdução}

O Design de Sistemas Produto-Serviço Sustentável (Sustainable Product Service+System - SPSS) apresenta-se como um modelo promissor na busca por novos modos de produção e consumo economicamente viáveis, socialmente justos e com baixo impacto ambiental (VEZZOLI et al., 2018). Nesse modelo, a inovação para sustentabilidade usualmente encontra-se além dos artefatos físicos, satisfazendo as necessidades dos usuários e stakeholders com respeito aos aspectos ambientais e sociais do entorno. Diversos autores corroboram que a adoção de um modelo de negócio de PSS ainda é uma atividade complexa (CAVALIERI, PEZZOTTA, 2012; MARQUES, 2018). A falta de métodos, técnicas e ferramentas que deem o suporte de forma adequada a esse processo criativo (considerando as múltiplas interações e individualidades dos sistemas, produtos e serviços) são alguns dos fatores que reforçam a esta complexidade (NGUYEN et al. 2014).

Alão (2020) destaca que a rápida e frequente mudança de comportamento dos usuários e aumento das conexões e da interdependência dos stakeholders envolvidos, também reforça a complexidade e relevância de se encontrar estratégias que visem acompanhar esse processo. Assim, coletar e interpretar informações, rapidamente e com assertividade, sobre os usuários e sobre os processos de produção, se tornou desafiador.

Nessa jornada para identificação de padrões, os grandes dados, conhecidos como big data, têm ganhado destaque, eles se transformam em ativos, empregando-se técnicas e métodos para analisá-los, para extrair informações relevantes (SPIEGEL, 2016). Esses dados são gerados por diferentes atores e dispositivos (gadgets), inclusive pelos próprios processos de manufatura e de experiência do usuário (SPIEGEL, 2016). Cabe ainda destacar que o desenvolvimento bem-sucedido de novos produtos e serviços dependem da efetiva transformação da informação em inteligência, passível de ser facilmente acessada e compartilhada na organização (MALHOTRA et al., 2007).

Ademais, a utilização da Informação por meio da análise de dados pode ajudar a organização a mudar a cultura de tomada de decisão, aumentando sua eficácia (FRISK, BANNISTER, 2017).

Assim, o presente artigo buscou compreender o estado da arte acerca da utilização do big data no Design, levando à seguinte pergunta: Quais são as ênfases, lacunas e eventuais contradições sobre a aplicação de big data no Design, e em particular de Sistemas Produto-Serviço Sustentáveis, disponíveis na literatura qualificada? 


\section{Big Data e Sistemas Produto-Serviço Sustentáveis}

À medida que os avanços tecnológicos mudam rapidamente, a fronteira entre as tarefas de trabalho realizadas por seres humanos e as realizadas por máquinas e algoritmos, também estão fazendo o mercado passar por grandes transformações (WEF, 2018), contexto do big data. Este pode ser definido por 5 características principais, os $5 \mathrm{Vs}: 1$ ) volume - grande quantidade; 2) velocidade - criados rapidamente e estão em movimento; 3) variedade - possuem diversas formas e surgem de diferentes fontes, podem ser ou não estruturados ou semiestruturados, 4) veracidade - em meio a tantos dados alguns podem ser incertos devido a sua inconsistência e incompletude, ambiguidades, entre outros, 5) valor - que o dado possui para quem o detém ou o pesquisa e é gerado pela interação das primeiras características (DEVI, 2019; MCAFEE, BRYNJOLFSSON, 2012; QUEIROZ, 2019).

O termo "data" ou "dados" é entendido neste trabalho como fatos, números, nomes, códigos, símbolos, datas, medidas, observações, palavras e outros itens desta natureza que estão fora de contexto (PRIOR, 2010).

\section{Processos e Tecnologias Relacionadas ao Big Data}

Alguns dos termos emergentes relacionados ao uso do big data que vêm sendo utilizados com mais frequência no âmbito do Design são: Data Science (DS), Business Analytics (BA), Data Mining (DM), Data Analysis (DA), Machine Learning (ML), Business Intelligence (BI), Internet das Coisas (Internet of Things - IoT) e Inteligência Artificial (Artificial Intelligence - AI) (PRIOR, 2019; FIALKOWSKI, SANTOS, 2019). O infográfico a seguir elucida algumas das principais interações relacionadas aos termos citados (Figura 1).

O big data é como um ecossistema, dentro do campo do data science (GIMENEZ et al., 2018). O data science é a ciência orientada a dados (BUFREM, 2016) e engloba preparação, limpeza e análise, de dados. Já o data analytics, envolve a aplicação de processos algorítmicos para analisar os dados.

A mineração de dados ou data mining, uma etapa anterior ao data analytics, pode ser considerada um conjunto de técnicas estatísticas e inteligência artificial. Sendo utilizada para encontrar relações, padrões e tendências anteriormente não identificados (PRIOR, 2010).

Todas essas novas tecnologias estão impactando diretamente no Design (OGG, 2019) e nas competências necessárias ao futuro profissional (WEF, 2018). Impactam também no processo de desenvolvimento de produtos, serviços ou sistemas (OGG, 2019). Assim, no contexto desta pesquisa, retoma-se a importância da junção de Design, e as novas tecnologias decorrentes do big data, a fim de guiar o processo de criação de novos Sistemas Produto+Serviço mais Sustentáveis. 
Fig 1. Correlação entre alguns dos principais conceitos e termos relacionados ao big data

Fonte: Os autores, 2020

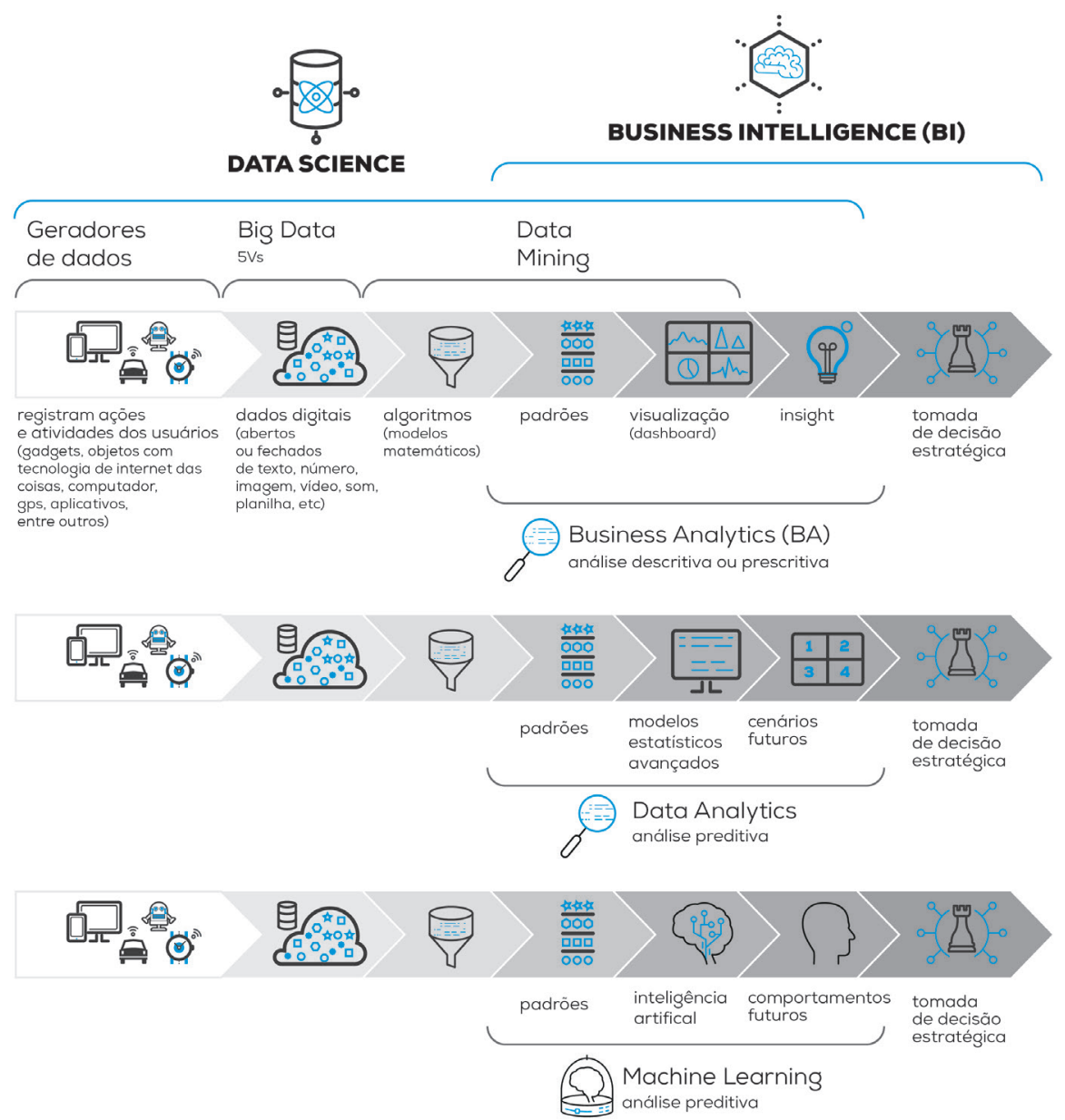

Design de PSS para a Sustentabilidade

Segundo Vezzoli et al. (2018) o conceito de Design orientado a prover forma a artefatos está ligado diretamente ao paradigma que considera a possibilidade do crescimento ilimitado da produção. Entretanto, a redução dos limites de resiliência do planeta tem demonstrado a inviabilidade deste (VEZZOLI et al., 2018, p.18). Uma das estratégias para reduzir ou mitigar os impactos do consumo consiste na aplicação dos métodos e técnicas associadas ao Design visando à sustentabilidade.

O Design para a Sustentabilidade pode ser um PSS Sustentável, que pode também ser definido como proposições de valor orientadas a satisfazer os usuários através da entrega de funções em vez de produtos (VEZZOLI et al., 2018; CESCHIN, 2016; COSTA JUNIOR, 2012; TUKKER, 2004). Neste contexto, a atividade do designer migra de projetista de artefatos para desenvolvedor de soluções que ofereçam bem-estar ao usuário sem perder os princípios de sustentabilidade (VEZZOLI et al., 2018; CESCHIN, 2016). 


\section{Impacto do Big Data para Soluções Sustentáveis em Design}

Corbett (2018) afirma que ao se definir sustentabilidade, a tomada de decisão deve levar em conta, simultaneamente, considerações econômicas, ambientais e sociais, estando inerentemente entrelaçada com o big data.

Ogg (2019) aponta que a integração das tecnologias digitais gerou novas possibilidades de reconfiguração da produção e do consumo. Assim, o foco do Design caminha para uma abordagem mais complexa e sistêmica onde o designer tem papel estratégico baseado na interação de pessoas e tecnologias. No entanto, em geral, as competências mínimas para os graduados em Design não contemplam estas novas práticas. O Design precisa responder a este contexto baseando suas ações na antecipação do futuro, prevendo a possibilidade de novas práticas habilitadas pelas tecnologias emergentes (OGG, 2019; WEF, 2018).

Dentre as competências propostas aos designers, destaca-se aqui também a questão ética, os limites legais e éticos do uso das tecnologias, compreendendo os riscos potenciais (OGG, 2019), já que este profissional pode ser considerado um agente "construtor de futuro" (FIALKOWSKI, 2019; CESCHIN, GAZIULUSOY, 2016; BHAMRA et al., 2011). Outra competência percebida é a capacidade de considerar os múltiplos agentes envolvidos, os sistemas complexos e a permeabilidade das áreas de fronteiras envolvidas nos processos de convergência de tecnologias. Assim, a habilidade multidisciplinar, com foco na aprendizagem ativa dos participantes, é imprescindível para gerar soluções com base no uso do big data (OGG, 2019; WEF, 2018).

Diante destes desafios, delineiam-se alguns temas importantes para reflexão quanto ao Design perante o uso do big data para PSS voltado à sustentabilidade: a tradução das abordagens emergentes decorrentes das novas tecnologias vistas para o universo das práxis do Design; as competências do Design para atuar neste novo contexto de complexidade maximizada; o desenvolvimento de estratégias e modelos para a viabilização do trabalho multidisciplinar; e a responsabilidade ética do Design nesse contexto.

\section{Método}

A presente pesquisa possui abordagem quantitativa, de caráter exploratório e com análise crítica da literatura, a sua natureza é descritiva. 0 método adotado para levantamento de dados foi inicialmente uma Revisão Bibliográfica Assistemática (RBA), seguida de uma Revisão Bibliográfica Sistemática (RBS).

A RBA realizada, além de permitir a estruturação da fundamentação teórica apresentada na seção anterior, identificou os principais temas e as possíveis palavras-chave para a procura sistemática. Já a RBS foi conduzida pelo roteiro proposto por Conforto et al. (2011) que possui etapas 
distribuídas em 3 fases (Entrada, Processamento e Saída). Para a fase de entrada, foram definidos o problema e objetivos, além das fontes primárias nas bases de Periódicos Capes, Science Direct e Scopus, por suas relevâncias e rigor de indexação. O primeiro grupo de termos (strings) selecionados relacionou-se à ciência de dados, sendo eles: big data, data mining, business intelligence e business analytics. O segundo grupo relaciona-se a Design Sustentável: Sustainable Product-Service System, Product-Service System, Service Design, Sustainable Design, Sustainable Consumption, Sustainable Development e Design. No processo de busca esses dois grupos de strings foram pesquisados de forma cruzada. Além disso, optou-se por utilizar apenas palavras em inglês pelo fato de os termos serem recentes e terem sido cunhados nessa língua, sendo assim usados mesmo quando o trabalho está na língua portuguesa.

\section{Resultados da RBS}

A partir de buscas nas bases foram identificados um total de 3.373 artigos revisados por pares. 0 maior volume de artigos foi resultante das strings "big data" AND "design" (429 artigos) e "data mining" AND "design" (1.323 artigos) (no Portal da Capes).

Após o Filtro Prévio, com a primeira exclusão de artigos de outros campos, 566 publicações passaram para o Filtro 1 (F1), destes foram lidos títulos, resumos e palavras-chaves, passando 41 publicações para o Filtro 2 (F2). No filtro 2 foram excluídos os arquivos duplicados, e então foram lidas as introduções e conclusões de 39 artigos. Finalmente, no último filtro (F3), os artigos foram lidos integralmente, resultando em 17 trabalhos.

Dos artigos selecionados, 2 trabalhos destacam-se por efetivamente conectarem big data ao desenvolvimento de PSS, mesmo sem contemplar a sustentabilidade. O primeiro, de Scherer et al. (2016), os autores atrelam o business analytics (BA) ao Design de PSS. Deste modo, por mais que o BA seja apenas um dos 10 itens dentro da metodologia proposta, este artigo é o que mais se aproxima da questão de pesquisa do presente estudo, porque os autores tratam do BA na etapa de Ideação de um PSS.

O segundo artigo, de Bertoni e Larsson (2017) apresenta-se uma revisão bibliográfica acerca do data mining e machine learning para o Design de PSS. O mesmo apresenta uma análise do resultado de 21 artigos que foram classificados conforme as principais contribuições para um projeto de PSS (BERTONI, LARSSON, 2017). Na sequência, os autores apresentam diversos questionamentos que podem ser feitos na fase de coletas de dados do data mining a fim de contribuírem com o PSS. Evidenciando, ao final, oportunidades e desafios das possíveis aplicações da mineração de dados em PSS. 
Dos 17 artigos, 10 visam à sustentabilidade, através da utilização do big data. Desses, só o artigo de Homberg e Sucha (2018) propõe uma estrutura para sustentabilidade ambiental. Por meio de um framework tem-se a finalidade de disponibilizar informações, além das oferecidas por órgãos do governo, para que empresas as aproveitem no desenvolvimento ou aprimoramento de seus serviços e produtos sustentáveis. $O$ artigo de Marwah et al. (2011) também trata exclusivamente dessa dimensão ao propor um modelo para avaliação automática do ciclo de vida de um produto, por meio de data mining.

Lock e Seele (2016) propõem que a sustentabilidade seja o centro de onde partem todos os stakeholders. Corbett (2017) fornece alguns exemplos de como o uso de big data pode proporcionar tomadas de decisão mais sustentáveis. Queiroz (2018) e Zhang et al. (2018) apresentam seus frameworks voltados para otimização de serviços de venda online. Já Zheng (2018), propõe um framework para gerar PSS orientado por dados, que ele chama de "Smart PSS". Shimomura (2018) propõe um método que emprega uma combinação de análise de tópicos por cluster para criar personas e construir cenários. Os artigos de Petrini e Pozzebon (2009), Muntean (2018) e Seele (2017), tratam da proposição de modelos para medir a sustentabilidade no âmbito empresarial. Dois artigos propõem uma reflexão ética quanto ao papel do designer ao utilizar o big data (GOODMAN, 2014; SEELE, 2017). Quanto aos trabalhos que propõe fórmulas matemáticas e não foram descartados, justificam-se pelos seguintes fatos: Li e Kauffman (2012) apresentam um mapa de jornada do usuário interligado com big data; e Dubey et al. (2018) apresentam um background teórico a respeito de big data atrelado às métricas.

Quanto à localização, tem-se uma ampla distribuição geográfica, sendo realizadas em Universidades da América do Norte, América do Sul, Ásia e uma concentração maior na Europa. Com relação ao período de publicação e quantidades de trabalhos vê-se que o campo de estudo é incipiente, dado que o critério de inclusão de trabalhos na RBS, no item data, foi de trabalhos publicados entre 2009 e 2019. A maior quantidade de artigos está nos últimos três anos, ressaltando-se a contemporaneidade do assunto e demonstrando um interesse crescente nos temas.

\section{Discussões}

A primeira discussão levantada a partir dos resultados é de que a utilização do big data no Design de Produto-Serviço Sustentável ainda não é expressiva (BERTONI, LARSSON, 2017; SCHERER et al., 2016; PETRINI, POZZEBON, 2009; MUNTEAN, 2018; SEELE, 2017; QUEIROZ, 2018). Apesar dos temas serem muito discutidos isoladamente, a junção de big data e PSS ainda é pouco encontrada (SCHERER et al., 2016; BERTONI, LARSSON, 
2017; ZHENG et al., 2018) e a junção de big data e sustentabilidade tampouco (SEELE, 2017; CORBETT, 2018). Ao juntar-se os três temas, tem-se uma redução ainda maior nos artigos encontrados (MUNIZ, SANTOS, 2015; FIALKOWSKI, SANTOS, 2019).

$\mathrm{Na}$ fundamentação teórica reforça-se ainda que, apesar do prospecto positivo destas novas tecnologias, o potencial das mesmas ainda não é bem explorado. Scherer et al. (2016) apontam que as TICs (Tecnologia da Informação e Comunicação) permitem acesso à diversos tipos de dados, mas as empresas em geral ainda não sabem como usá-los para obter vantagens, nem como utilizá-los para a ideação de PSSs. Apesar das empresas serem capazes de coletar quantidades imensas de dados, o uso dos mesmos está limitado às questões de manutenção e gestão, poucas vezes os dados são usados para ideação em Design (PETRINI, POZZEBON, 2009; BERTONI, LARSSON, 2017).

Nessa mesma discussão encontra-se o trabalho de Seele (2017), que analisa os primeiros esforços para utilizar big data para promover a sustentabilidade e estimular políticas de prevenção de danos relacionados às questões ambientais e sociais. Assim, é sugerido o potencial de uso dos algoritmos preditivos, que são usados hoje para prever o comportamento do consumidor e mapear decisões de compra, prevendo também eventos futuros relacionados à sustentabilidade. Corbett (2018) corrobora essa visão, colocando que apesar do autor entender a relação intrínseca do big data com a Sustentabilidade, quando se considera a sustentabilidade como um meio, também se toma decisões levando em conta aspectos econômicos, ambientais e sociais, mas não se olha para as possíveis repercussões e danos que esta relação pode trazer para a sustentabilidade.

Diversos autores apontam a qualidade e rapidez ao gerar informações e obter valor a partir do big data (HOMBERG, SUSHA, 2018; DUBEY et al., 2018; SEELE, 2017; CORBETT, 2018), alinhando-se com o que foi visto no levantamento teórico (LÓPEZ-ROBLES et al., 2019; FIALKOWSKI, SANTOS, 2019). A esse respeito, Scherer et al. (2016) apontam que a partir da análise dos dados, o comportamento do consumidor e as percepções do mercado podem ser avaliadas em tempo real e assim identificam-se oportunidades para inovar. O trabalho de Seele (2017) aponta que o uso de dados, como tecnologias de machine learning, data mining e IoT, permitem uma formação científica baseada na ciência e orientada para o objetivo, permitindo uma maior velocidade e assertividade no processo de predição que por meios tradicionais. 
Classificação dos Artigos Considerando os Temas Big Data, Sustentabilidade e Design

A RBS levantou algumas definições e teorias a respeito dos temas que se interseccionam neste trabalho. Assim, o Figura 2 evidência como os resultados se comportam perante a base teórica prévia.
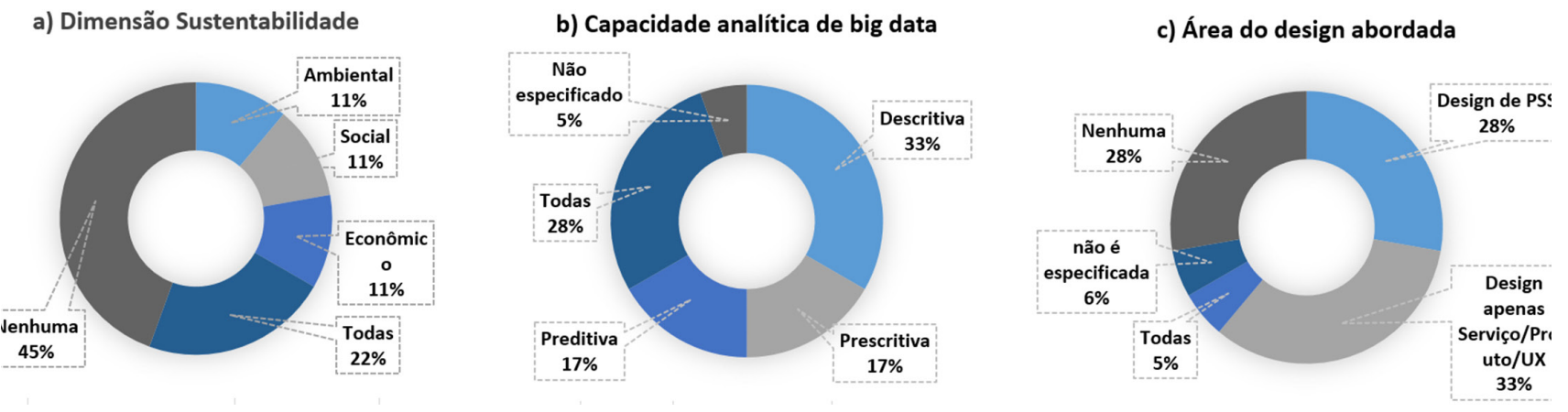

Fig 2. Classificação dos trabalhos finais da RBS

Fonte: Os autores, 2020

A Figura 2(a) permite apontar que $45 \%$ dos trabalhos simplesmente não focam na sustentabilidade, mesmo o tema tendo feito parte das strings de busca. Ainda no gráfico 1(a), observa-se que os artigos que tratam da sustentabilidade, abordam em sua maioria todas as três dimensões (SEELE, 2017; CORBETT, 2018). No uso das strings ligadas a sustentabilidade e PSS, a maior parte dos artigos não trata exatamente da união dos temas (SCHERER et al., 2016; BERTONI, LARSSON, 2017; GOODMAN, 2014; MUNTEAN, 2018; ZHENG, 2018; SHIMOMURA, 2018; CORBETT, 2018). Assim entende-se aqui também, novamente, a lacuna de que os temas relacionados entre si (sustentabilidade com PSS e big data) não são muito presentes na literatura. 0 Gráfico 1(b) também mostra, quanto à capacidade analítica do big data, que a maior parte dos artigos abordam as análises descritivas e prescritivas, a análise preditiva, que é a forma mais sofisticada e complexa de analytics, não foi constante na literatura levantada. Apenas os artigos de Seele (2017), Zhang (2018) e Dubey (2018) falam especificamente do assunto, demonstra-se assim outra lacuna.

A área do Design (c) mais abordada na pesquisa realizada deveria ser a de PSS, uma vez que as strings de busca contemplavam este termo. Porém, a busca dirigida ainda trouxe diversos artigos que não ficavam nessa área do Design e sim exclusivamente em Design de produto (MARWAH, 2011; ZHANG, 2018), ou apenas serviço (LI, KAUFFMAN, 2012) ou ainda só UX Design (GOODMAN, 2014), ou simplesmente não falavam de Design (PETRINI, POZZEBON, 2009; MUNTEAN, 2018; SEELE, 2017; CORBETT, 2018; DUBEY, 2018). Dos artigos que falam especificamente de PSS é importante citar 
aqui o trabalho de Scherer et al. (2016) e de Bertoni e Larsson (2017), porque ambos destacam o PSS orientado ao uso e ao resultado, no qual os usuários não detêm a propriedade de artefatos físicos, mas garantem a satisfação que se espera no resultado final da entrega.

Além disso, a Figura 2 também evidencia que, apesar de diversos pesquisadores enxergarem os Sistema de Serviços (PSS) como uma abordagem promissora para a sustentabilidade (VEZZOLI et al., 2018; CESCHIN, 2016; COSTA JUNIOR, 2012; TUKKER, 2004), o resultado da RBS demonstrou que nem sempre os autores têm o foco em sustentabilidade ao propor os PSSs (SCHERER et al., 2016; BERTONI, LARSSON, 2017; GOODMAN, 2014; MUNTEAN, 2018; ZHENG, 2018; SHIMOMURA, 2018).

\section{Cruzamento da Literatura Encontrada na RBA e RBS}

Para evidenciar os temas de interesse levantados na fundamentação teórica por meio da RBA, perante as lentes do PSS e sustentabilidade, os mesmos foram contrapostos aos artigos levantados na RBS, e serão discutidos a seguir, com o apoio do Gráfico 2. Os temas foram os seguintes: a) A tradução das abordagens emergentes decorrentes das novas tecnologias vistas para o universo da práxis do Design ("Tecnologia" no Gráfico); b) As competências do Design para atuar neste novo contexto de complexidade maximizada ("Competências"); c) 0 desenvolvimento de estratégias e modelos para a viabilização do trabalho multidisciplinar ("Interdisciplinaridade"); d) A responsabilidade ética ("Ética"). Cada um desses temas recebeu uma nota de acordo com a forma com que foi abordado em cada artigo, sendo que 0 (zero) significa que o tema não foi tratado no respectivo trabalho, 1 (um) o tema foi tratado de maneira genérica e 2 (dois) o tema foi bem explorado no respectivo artigo.

Fig 3. Classificação dos trabalhos finais da RBS conforme os principais temas Fonte: Os autores, 2020

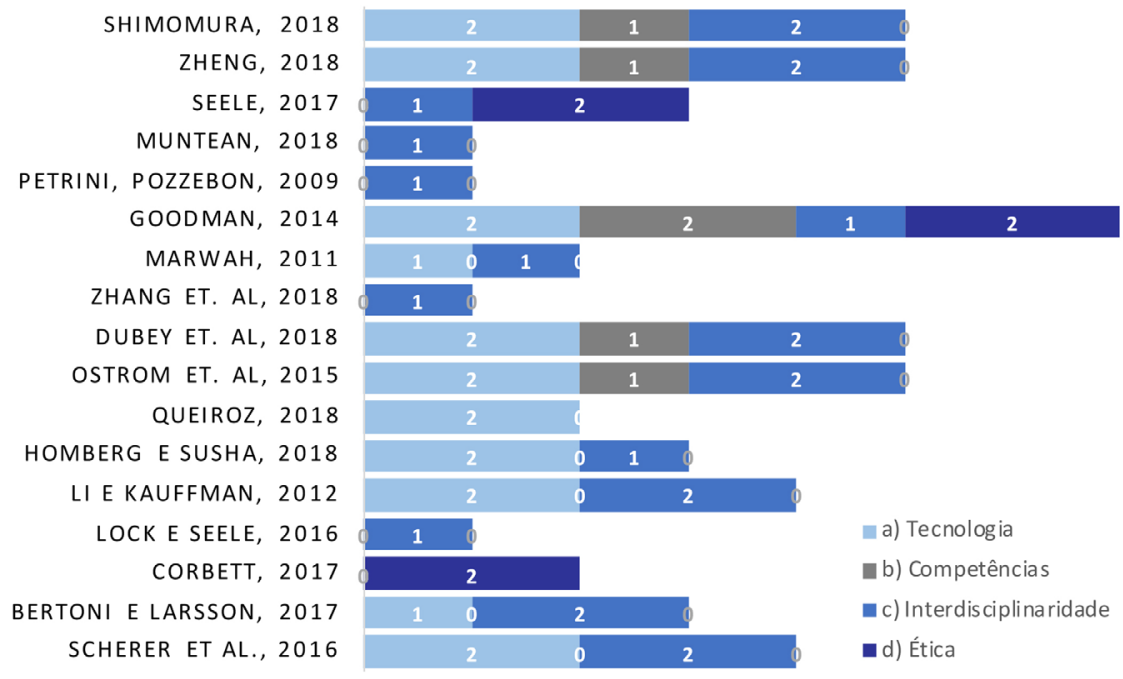


A Figura 3 destaca que o artigo de Goodman (2014) é o mais completo quanto aos temas de interesse deste trabalho, porque obtem a maior pontuação e trata dos 4 temas. Os artigos de Shimomura (2018), Zheng (2018), Dubey et al. (2018) e Ostrom et al. (2015), também se destacam. Os trabalhos de Zhang et al. (2018) e de Lock e Seele (2016), apesar de terem sido selecionados e serem interessantes no que tange as dimensões aqui tratadas, obtiveram as mais baixas pontuações. Nota-se também que os temas tecnologia e interdisciplinaridade são constantes em todos os trabalhos, demonstrando ênfase. Enquanto o tema competências e ética, não são constantes, podendo representar assuntos mais recentes na literatura.

Pode-se também afirmar que quanto à tradução das abordagens emergentes decorrentes das novas tecnologias vistas para o universo das práxis do Design (a) diversos autores (BERTONI, LARSSON, 2017; ZHENG et al., 2018; ZHANG, 2018) levantam a questão de que o processo de Design deve ser repensado de maneira que formalize o uso dos dados nos desenvolvimentos de produtos e serviços (ênfase). Em especial a pesquisa de Bertoni e Larsson (2017) evidencia que nenhum método de PSS encontrado pelos autores evidencia o foco exclusivo neste modelo de sistema, constituindo-se apenas de adaptações no processo de desenvolvimento de Design de produto, apesar do alto grau de complexidade quando se trata de sistemas com serviços. Goodman (2017) frisa que o processo do uso de big data para Design de serviços também não contempla uma mediação ética. Por conseguinte, a RBS feita enfatizou a lacuna de não haver métodos que integrem a perspectiva dos serviços, quando são usados inputs de data science para planejar o PSS. Já para Shimomura et al. (2018), a preocupação quanto ao método de PSS é que o mesmo não prevê solução adequada para os diversos stakeholders, em especial à diversidade de usuários, envolvidos em um sistema de PSS. Então os autores sugerem a criação de personas e cenários com base em big data, para entender possíveis requisitos necessários a esses usuários, defendendo que este método é superior à análise demográfica simples, normalmente utilizada.

Petrini e Pozzebon (2009), Muntean (2018) e Seele (2017) também afirmam que falta um processo claro no uso de dados para dar suporte e monitorar objetivos de sustentabilidade. Defendem que este processo deve estar integrado aos objetivos financeiros da empresa e na sua estratégia. Dubey et al. (2018) argumentam que o big data provê oportunidades únicas às organizações para melhorarem a visibilidade e a integração em redes de cadeia de suprimento com vista à produção e consumo sustentáveis.

Quanto às competências do Designer para atuar neste novo contexto de complexidade maximizada (b), muitos autores corroboram com o que foi visto na fundamentação, afirmando que existe um gap entre as competências dos profissionais envolvidos em data science e as competências do designer para lidar com dados (WEF, 2018; OGG, 2019; SCHERER et al., 2016; BERTONI, LARSSON, 2017). Assim é notável a necessidade do Designer se envolver mais no mundo dos dados, de maneira a trazer contribuições no 
Design de PSS (ênfase). Goodman (2017) e Corbett (2018) sugerem que os designers não estão preparados e devem se capacitar para uma política de ética de dados. Goodman (2017) aponta que diversos tipos de dispositivos de Design de interação que, ao ser centrado no usuário, deve ter fácil acesso aos dados pessoais que, por sua vez, podem ser usados para prejudicar o consumidor, mesmo que de maneira velada.

Ainda na Figura 3, quanto ao desenvolvimento de estratégias e modelos para a viabilização do trabalho interdisciplinar (c), alguns autores apontam que a natureza do Design e o trabalho em paralelo com diversos times tornam esta tarefa mais complexa (BERTONI, LARSSON, 2017; ZHANG, 2018). No entanto, tal integração é essencial e deve ser empregada, potencializando o sucesso de desenvolvimento de PSSs mais sustentáveis.

Por fim, quanto à responsabilidade ética vista na fundamentação (d) (FIALKOWSKI, 2019; CESCHIN, GAZIULUSOY, 2016; BHAMRA et al., 2011), nota-se que apenas 3 artigos abordam o tema (GOODMAN, 2014; SEELE, 2017; CORBETT, 2018) e que apenas o trabalho de Goodman (2014) trata especificamente deste assunto na íntegra. Segundo SEELE (2017) a previsão de futuros por meio do uso de dados de pessoas reais pode representar uma violação das normas existentes. As preocupações éticas em relação ao uso de dados, para Goodman (2014), emergem de quatro tendências/tecnologias que a autora identifica: todo o tipo de sensores coletando dados em tempo real; os dados sendo usados como commodities, podem ser estocados e reutilizados indefinidamente; a falta de transparência de quem está "por trás das cortinas" dos dados; e a escala em massiva dos dados, que invade e sobrepõe questões culturais. Assim os designers devem refletir sobre como levar em conta diferentes expectativas, que devem ser culturalmente flexionadas para a coleta e reutilização de dados e assim evitar arrependimentos éticos. Assim, demonstra-se uma ênfase na preocupação com o assunto, mas que ainda é uma lacuna a ser explorada.

\section{Conclusão}

Com o levantamento do estado da arte por meio da RBA e RBS, foram demonstradas as intersecções teóricas entre sistemas de inteligência, no qual se insere o big data, e a sustentabilidade, sob a ótica do Design (PSS). Observou-se assim que, apesar do aumento substancial do número de estudos nos temas referentes à 'PSS' e 'big data', quando estes termos são pesquisados de forma conjunta, são praticamente ausentes (PETRINI, POZZEBON, 2009; PETRINNI, 2006; FIALKOWSKI, SANTOS, 2019).

Outras lacunas principais apresentadas na pesquisa referem-se: a falta de convergência quanto ao uso dos termos e nomes relacionados ao big data, o baixo índice do uso dos dados para análises preditivas em Design e PSS sendo tratado na literatura sem o foco da sustentabilidade. Dentre as principais lacunas, também está à falta de competências do designer para lidar com o uso de dados e com as rápidas mudanças tecnológicas. Assim é 
fundamental que as empresas tenham um papel ativo no apoio a sua força de trabalho por meio do desenvolvimento de competências e qualificações. De forma particular, interessa que indivíduos adotem uma abordagem proativa para a própria aprendizagem, e que os governos também criem um ambiente favorável para apoiar esses esforços.

Ainda quanto às lacunas, é importante salientar que a tradução das abordagens emergentes decorrentes das novas tecnologias vistas para o universo das práxis do Design, deve ir além do desenvolvimento das competências, deve ser pautado também por mudanças no processo. Assim, falta estabelecer um processo claro relativo ao desenvolvimento de PSS, usando big data para dar suporte e monitorar os objetivos de sustentabilidade. Enfatiza-se neste contexto o entendimento dos autores referentes à necessidade de produção acadêmica, e desenvolvimento de estratégias e modelos para a viabilização do trabalho interdisciplinar do designer. Alguns autores enfatizam ainda que a ideação de PSSs Sustentáveis possuem características complexas que podem ser suportadas pela utilização inteligente e adequada do big data. No entanto a questão ética no uso de dados em Design de PSS também é uma deficiência na literatura porque há pouca discussão a respeito, embora a demanda por uma política de uso de dados seja necessária e observada por alguns autores.

Perante tais lacunas vistas, um possível desdobramento deste estudo seria a investigação do processo de desenvolvimento de Design, PSS e do uso dos dados, assim podendo sugerir diretrizes este processo.

A contribuição aqui apresentada está delimitada ao seu aspecto teórico. Diante do exposto, também se recomendam futuras pesquisas que possam elucidar no campo prático ou empírico desses mesmos temas.

Agradecimento

Este estudo foi parcialmente financiado pela Coordenação de Aperfeiçoamento de Pessoal de Nível Superior Brasil (CAPES) - Código Financeiro 001.

\section{Referências}

ALÃO, Rui SD. Sobre a complexidade dos problemas contemporâneos de design. DAT Journal, v. 5, n. 4, p. 24-40, 2020.

BERTONI, A.l.; LARSSON, T. Data Mining in Product Service Systems Design: Literature Review and Research Questions. The 9th CIRP IPSS Conference: Circular Perspectives on Product/Service-Systems, v. 64, p. 306-311, 2017.

BHAMRA, T.; LILLEY, D.; TANG, T. Design for sustainable behaviour: Using products to change consumer behaviour. The Design Journal, v. 14, n. 4, p. 427-445, 2011.

BRITTO, F. P. Perspectivas organizacional e tecnológica da aplicação de analytics nas organizações. 2016. 95 f. Dissertação (Mestrado em Tecnologias da Inteligência e Design Digital) Pontifícia Universidade Católica de São Paulo, São Paulo, 2016. 
BUFREM, L. S. et al. Produção Internacional Sobre Ciência Orientada a Dados: análise dos termos Data Science e E-Science na Scopus e na Web of Science. Informação \& Informação, v. 21, n. 2, p. 40-67, 2016.

CESCHIN, F.; GAZIULUSOY, I. Evolution of design for sustainability: From product design to design for system innovations and transitions. Design studies, v. 47, p. 118-163, 2016.

CORBETT, C. J. How Sustainable Is Big Data? Production and Operations Management. v. 27, n. 9, p. 1685-1695, 2018.

CONFORTO, E. C. et al. Roteiro para revisão bibliográfica sistemática: aplicação no desenvolvimento de produtos e gerenciamento de projetos. In: $8^{\circ}$ CONGRESSO BRASILEIRO DE GESTÃO DE DESENVOLVIMENTO DE PRODUTOS - CBGDP. Anais p. 1- 12, Porto Alegre, 2011.

COSTA JUNIOR, J. da. Proposição de um Modelo de Referência para o Design de Serviços Ecoeficientes em Sistemas Produto-Serviço. 201f. Dissertação: Programa de Pós-Graduação em Design - Universidade Federal do Paraná, Curitiba, 2012.

DEVI, G. N. R. Emerging Trends in Big Data Analytics - A Study. In: Kumar A., Mozar S. (eds) ICCCE 2018. ICCCE 2018. Lecture Notes in Electrical Engineering, v.500, p. 563-570. Springer, Singapore, 2019.

DUBEY, R. et al. Examining the role of big data and predictive analytics on collaborative performance in context to sustainable consumption and production behavior. Journal of Cleaner Production, v. 196, p. 1508-1521, 2018.

FIALKOWSKI, V. P. Prospecção e Gestão de Design: Busca da Inovação Guiada pelo Significado. 296f. Dissertação: Programa de Pós-Graduação em Design - Universidade Federal do Paraná, Curitiba, 2019.

FIALKOWSKI, V. P.; SANTOS, A. Design para o Comportamento Sustentável: Cocriação de Mapas Conceituais. MIX Sustentável, v. 5, n. 5, p. 19-30, dez. 2019.

FRISK, J. El.; BANNISTER, F. Improving the use of analytics and big data by changing the decision-making culture: A design approach. Management Decision, v. 55, e.10, p. 2074-2088, 2017. GOODMAN, E. Design and ethics in the era of big data. Interactions, v. 21, n. 3, p. 22-24, 2014. HOMBERG, M. V. D.; SUSHA, I. Characterizing Data Ecosystems to Support Official Statistics with Open Mapping Data for Reporting on Sustainable Development Goals. International Journal of Geo-Information, v. 7, n. 12, 2018.

LI, T.; KAUFFMAN, R. J. Adaptive learning in service operations. Decision Support Systems, v. 53, e. 2, p. 306-319, 2012.

LOCK, I.; SEELE, P. Theorizing stakeholders of sustainability in the digital age. Sustainability Science, v. 12, n. 2, p. 235-245, 2017.

LÓPEZ-ROBLES, J. R., OTEGI-OLASO, J. R.; GÓMEZ, I. P.; COBO, M. J. 30 years of intelligence models in management and business: A bibliometric review. International Journal of Information Management, v. 48, p. 22-38, 2019.

MALHOTRA, N. K.; KIM, S. S.; PATIL, A. Common method variance in IS research: A comparison of alternative approaches and a reanalysis of past research. Management science, v. 52, n. 12, p. 1865-1883, 2006.

MANZINI, E.; VEZZOLI, C. A. O desenvolvimento de produtos sustentáveis. Os requisitos ambientais dos produtos industriais. Edusp, 2002.

MARWAH, M.; SHAH, A.; BASH, C.; PATEL, C.; RAMAKRISHNAN, N. Using data mining to help design sustainable products. Computer, v. 44, n. 8, p. 103-106, 2011.

MATTOS, P. C. Tipos de Revisão de Literatura. Faculdade de ciências agronômicas: UNESP, Batucatu, 2015. 
MCAFEE, A.; BRYNJOLFSSON, E. Big data: the management revolution. Harvard Business Review. 90 (10), p. 60-68, 2012.

MUNTEAN, M. Business intelligence issues for sustainability projects. Sustainability, v. 10, n. 2, p. 335, 2018.

MUNIZ, M. O.; SANTOS, A. D. A Pesquisa em Design para o Comportamento Sustentável: Lacunas e Desafios. Mix sustentável, v. 1, n. 2, p. 58-67, 2015.

OGG, C. M. N. G. Modelo para estabelecer competências para o futuro do design orientado pelas tecnologias emergentes. $339 \mathrm{f}$. Tese (Doutorado em Design) - Setor de Artes, Comunicação e Design da Universidade Federal do Paraná, Curitiba, 2019.

OSTROM, A. L.; PARASURAMAN, A; BOWEN, D. E.; PATRÍCIO, L.; VOSS, C. A. Service Research Priorities in a Rapidly Changing Context. Journal of Service Research, v. 18, n. 2, p. 127-159, 2015. PERES, R. S.; DE SÁ, N. O. Ferramentas web 2.0 em unidades de informação: aspectos de divulgação e melhora contínua identificados em artigos científicos. Informação \& Informação, v. 24, n. 2, p. 263-286, 2019.

PETRINI, M.; POZZEBON, M. Managing sustainability with the support of business intelligence: Integrating socio-environmental indicators and organisational context. The Journal of Strategic Information Systems, v. 18, n. 4, p. 178-191, 2009.

PIRES, R. D. L. Implantação de ferramenta de análise preditiva: um estudo de caso. 2017. 89 f. Dissertação (mestrado) - Escola de Administração de Empresas de São Paulo da Fundação Getúlio Vargas, São Paulo, 2017.

PRIOR, V. Glossary of terms used in competitive intelligence and knowledge management. Virginia: SCIP-Strategic and Competitive Intelligence Professionals, 2010.

QUEIROZ, M. M. A framework based on Twitter and big data analytics to enhance sustainability performance. Environ Qual Manage, v. 28, n. 1, p. 95-100, 2018.

SCHERER, J. O.; KLOECKNER, A. P.; RIBEIRO, J. L. D.; PEZZOTTA, G.; PIROLA, F. Product-Service System (PSS) design: Using Design Thinking and Business Analytics to improve PSS Design. Procedia CIRP, v. 47, p. 341-346, 2016.

SEELE, P. Predictive sustainability control: A review assessing the potential to transfer big data driven 'predictive policing' to corporate sustainability management. Journal of cleaner production, v. 153, p. 673-686, 2017.

SHIMOMURA, Y.; NEMOTO, Y.; ISHII, T.; NAKAMURA, T. A method for identifying customer orientations and requirements for product-service systems design. International Journal of Production Research, v. 56, n. 7, p. 2585-2595, 2018.

SPIEGEL, R. Home Big Data and Cloud Computing Have Moved into Design. Design News. v. 71, e. 10, p. 38-39, 2016. < https://www.designnews.com/automation-motion-control/big-data-and-cloud-computing-have-moved-design/201803187344987> Acesso em 13.maio.2019. TUKKER, A. Eight types of product-service system: eight ways to sustainability? Experiences from SusProNet. Business strategy and the environment, v. 13, n. 4, p. 246-260, 2004.

VEZZOLI, C.; KOHTALA, C.; SRINIVASAN, A.; DOS SANTOS, A.; XIN, L.; CHAVES, L. I.; ...; ENGLER, R. C. Sistema Produto+ Serviço Sustentável: Fundamentos. 2018. Tradução de Aguinaldo dos Santos. Curitiba, Insight, 2018.

WEF - WORLD ECONOMIC FORUM. The Future of Jobs Report. In: Global Challenge Insight Report, World Economic Forum, Geneva. 2018. Acessado em 27 de junho de 2019 <http:// www3.weforum.org/docs/WEF_Future_of_Jobs_2018.pdf>.

Recebido: 05 de janeiro de 2021. Aprovado: 10 de fevereiro de 2021.
ZHANG, J. et al. Product features characterization and customers' preferences prediction based on purchasing data. CIRP Annals - Manufacturing Technology, v. 67, n. 1, p. 149-152, 2018. 